\title{
Acute effects of difference in glucose intake on arterial stiffness in healthy subjects
}

\author{
Ryota Kobayashi ${ }^{1}$, Kaori Sato ${ }^{2}$, Miki Sakazaki ${ }^{3}$, Yukie Nagai ${ }^{3}$, \\ Soichiro Iwanuma ${ }^{4}$, Nobuyuki Ohashi ${ }^{4}$, Takeo Hashiguchi ${ }^{4}$ \\ ${ }^{1}$ Center for Fundamental Education, Teikyo University of Science: 2-2-1 Senju, \\ Sakuragi, Adachi-ku, Tokyo, Japan \\ ${ }^{2}$ College of Liberal Arts, International Christian University: 3-10-2, Osawa, Mitaka Shi, Tokyo To, Japan \\ ${ }^{3}$ Research and Development Division, Mitsui Sugar Co., Ltd: 36-2 Nihonbashihakozakicho, \\ Chuo City, Tokyo, Japan \\ ${ }^{4}$ Department of School Education, Teikyo University of Science: 2-2-1 Senju, \\ Sakuragi, Adachi-ku, Tokyo, Japan
}

\begin{abstract}
Background: Post-prandial hyperglycemia is associated with higher cardiovascular risk, which causes arterial stiffening and impaired function. Although post-prandial increases in blood glucose are proportional to the level of intake, the acute effects of different glucose intakes on arterial stiffness have not been fully characterized. The present study aimed to determine the acute effects of differences in glucose intake on arterial stiffness.

Methods: Six healthy middle-aged and elderly individuals (mean age, $60.0 \pm 12.1$ years) were orally administered 15, 20, and $25 \mathrm{~g}$ of glucose on separate days in a randomized, controlled, cross-over fashion. Brachial-ankle pulse wave velocity, heart-brachial pulse wave velocity, cardio-ankle vascular index, brachial and ankle blood pressure, heart rate, and blood glucose and serum insulin concentrations before and 30, 60, and 90 min after glucose ingestion were measured.

Results: Compared to baseline, brachial-ankle pulse wave velocity was higher at 30, 60 and 90 min after ingestion of $25 \mathrm{~g}$ glucose, and higher at $90 \mathrm{~min}$ after ingestion of $20 \mathrm{~g}$ glucose, but at no time points after ingestion of $15 \mathrm{~g}$. Cardio-ankle vascular index was higher at 60 min than at baseline after ingestion of $25 \mathrm{~g}$ glucose, but not after ingestion of 15 or $20 \mathrm{~g}$.

Conclusions: These results suggest that brachial-ankle pulse wave velocity and cardio-ankle vascular index is affected by the quantity of glucose ingested. Proposed presently is that glucose intake should be reduced at each meal to avoid increases in brachial-ankle pulse wave velocity and cardio-ankle vascular index during acute hyperglycemia. (Cardiol J 2021; 28, 3: 446-452)
\end{abstract}

Key words: arterial stiffness, brachial-ankle pulse wave velocity, cardio-ankle vascular index, quantity of glucose ingestion, healthy subjects

\section{Introduction}

High central (aortic), peripheral (leg) and systemic pulse wave velocity (PWV) and cardio-ankle vascular index (CAVI) are indices of arterial stiffness and are used as important determinants and indices of cardiovascular risk [1,2]. Moreover, high ankle systolic blood pressure (SBP) has been proposed as an independent risk factor for the development of cardiovascular disease [3]. Wohlfahrt et al. [4] reported that arterial stiffness correlates with ankle SBP. Inhibition of any increases in arte-

Address for correspondence: Ryota Kobayashi, PhD, Center for Fundamental Education, Teikyo University of Science:

2-2-1 Senju, Sakuragi, Adachi-ku, Tokyo 120-0045, Japan, tel: +81 809193 3605, fax: 03-6910-3800,

e-mail: rkobayashi@ntu.ac.jp

Received: 7.05.2019

Accepted: 29.09.2019

This article is available in open access under Creative Common Attribution-Non-Commercial-No Derivatives 4.0 International (CC BY-NC-ND 4.0) license, allowing to download articles and share them with others as long as they credit the authors and the publisher, but without permission to change them in any way or use them commercially. 
rial stiffness and ankle SBP might thus be beneficial for health.

Arterial stiffness and ankle SBP acutely increase after glucose ingestion [5, 6], and higher post-prandial hyperglycemia is associated with cardiovascular risk, which causes arterial stiffening and impaired functioning [7]. Furthermore, a new World Health Organization (WHO) guideline recommends that adults and children reduce the daily intake of free sugars to $<25 \mathrm{~g}$ [8]. Although post-prandial increases in blood glucose (BG) are known to be proportional to the level of intake [9], whether arterial stiffness and SBP will increase after ingesting $<25 \mathrm{~g}$ of glucose remains unclear.

The present study aimed to investigate the acute effects of differences in glucose intake on arterial stiffness and SBP. Hypothesized herein is that differences in glucose intake would affect changes in arterial stiffness and SBP after glucose ingestion in older adults.

\section{Methods}

\section{Subjects}

In this study there are 6 healthy subjects (mean age, $60 \pm 12$ years). All lived a sedentary lifestyle ( $\geq 2$ years without regular exercise as assessed by the international physical activity questionnaire) and were normotensive $(<140 / 90$ $\mathrm{mmHg}$ ), normoglycemia ( $<109 \mathrm{mg} / \mathrm{dL}$ ), nonsmokers, without symptoms or history of overt chronic disease (Table 1). All participants were fully informed about the experimental procedures as well as the purpose of the study before providing written, informed consent to participate. The Ethics Committee at Teikyo University of Science approved this study, which proceeded in accordance with the guidelines for human experimentation published by the documented institutional review board (approval no. 18034). This study also conformed to the principles of the Declaration of Helsinki.

\section{Study design}

Each participant completed 3 trials in random order: orally administered 15, 20, and $25 \mathrm{~g}$ glucose (in $200 \mathrm{~mL}$ of water, consumed within $5 \mathrm{~min}$ ) on separate days. All subjects then rested for at least $10 \mathrm{~min}$ in a supine position to establish a stable baseline. Each volunteer waited about 2-3 days after completion of one trial before crossing over to the next trial. Brachial-ankle PWV was measured, (baPWV indicative of systemic arterial stiffness), heart-brachial PWV (hbPWV, indicative of proximal aortic arterial stiffness), brachial and
Table 1. Characteristics of study participants.

\begin{tabular}{lc}
\hline Parameters & Value \\
\hline Age [years] & $60 \pm 12$ \\
Height [cm] & $165 \pm 11$ \\
Weight $[\mathrm{kg}]$ & $62 \pm 14$ \\
Body fat [\%] & $24 \pm 4$ \\
BMI [kg/m²] & $22 \pm 2$ \\
Brachial SBP [mmHg] & $120 \pm 20$ \\
Ankle SBP [mmHg] & $141 \pm 11$ \\
BG [mg/dL] & $94 \pm 9$ \\
Insulin [mg/dL] & $5 \pm 3$ \\
HDL-C [mg/dL] & $58 \pm 7$ \\
Triglycerides [mg/dL] & $97 \pm 25$ \\
\hline
\end{tabular}

Values represent mean \pm standard deviation; $\mathrm{BMI}$ - body mass index; BG — blood glucose; HDL-C — high-density lipoprotein cholesterol; SBP - systolic blood pressure

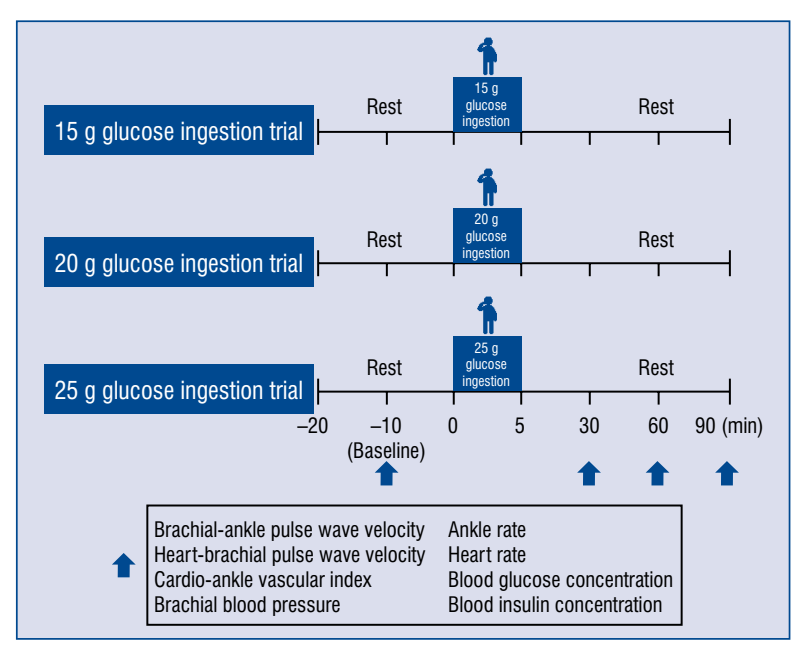

Figure 1. Study design. After $10 \mathrm{~min}$ of supine rest, pulse wave velocity, cardio-ankle vascular index, brachial and ankle blood pressure, heart rate, blood glucose level and blood insulin level were measured before (baseline) and at 30,60 and 90 min after glucose ingestion $(15,20$ and $25 \mathrm{~g})$.

ankle SBP, diastolic blood pressure (DBP), pulse pressure (PP), heart rate (HR), CAVI (indicative of systemic arterial stiffness), and BG and serum insulin concentrations, before (baseline) and 30, 60, and $90 \mathrm{~min}$ after glucose ingestion. All these measurements, including baPWV, hbPWV, CAVI, and fasting BG, were evaluated after $12 \mathrm{~h}$ of fasting (Fig. 1).

\section{Anthropometrics}

Height and weight were measured to the nearest $0.5 \mathrm{~cm}$ using a stadiometer, and body composi- 
tion was determined using a body composition analyzer (WB-150 PMA; TANITA Co., Tokyo, Japan).

\section{Arterial stiffness}

baPWV, hbPWV, BP, and HR was measured using a form PWV/ABI vascular testing device [10] (Fukuda-Colin Co., Tokyo, Japan) and CAVI using a VaSera VS- $1500^{\circledast}$ vascular testing device [11] (Fukuda Denshi Co., Tokyo, Japan). baPWV is an index that reflects systemic arterial stiffness and hbPWV reflects proximal aortic stiffness. The CAVI is an index that reflects systemic arterial stiffness is theoretically adjusted by blood pressure.

\section{Blood pressure and heart rate}

Brachial and ankle SBP, mean BP (MBP) and DBP, PP and HR while in supine resting were measured in triplicate using an automated oscillometric-form PWV/ABI device (Form PWV/ /ABI; Fukuda-Colin Co., Tokyo, Japan) over the brachial and ankle arteries. Carotid SBP, which has been proposed as an indicator of the magnitude of wave reflections, was also obtained from pressure waveforms [12].

\section{Blood glucose and insulin levels}

Venous blood was withdrawn from participants via catheter in the right or left arm before, and at 30,60 and $90 \mathrm{~min}$ after ingestion of 15, 20 or $25 \mathrm{~g}$ glucose. Levels of BG and insulin were assayed using the ultraviolet-hexokinase method and chemiluminescent enzyme immunoassay, respectively.

\section{Oral glucose ingestion}

15,20 , and $25 \mathrm{~g}$ of glucose were orally administered to each participant (in $200 \mathrm{~mL}$ of water, consumed within $5 \mathrm{~min}$ ) on separate days using a cross-over design in the morning after an overnight $(12 \mathrm{~h})$ fast. The glucose beverage $(200 \mathrm{~mL})$ was within the standard for adult humans and was consumed within 5 min [13].

\section{Sample size}

A power analysis was performed with $G *$ Power 3 to determine the appropriate sample size. Effect size for arterial stiffness at the laboratory was 0.5 . To detect differences with $60 \%$ power and a one-tailed $\alpha$ of $5 \%$ in the laboratory using analysis of variance, it was calculated that each intervention would require 6 individuals.

\section{Statistical analyses}

All data are presented as mean \pm standard deviation (SD). The normal distribution of all data was confirmed using the Kolmogorov-Smirnov test. Data were analyzed using repeated-measures two-way analysis of variance, with variables of group and time. Significant differences between mean values were identified using the Bonferroni post-test. Data were analyzed using SPSS version 25 (IBM, Armonk, NY), with statistical significance being accepted at the level of $\mathrm{p}<0.05$.

\section{Results}

Baseline baPWV, hbPWV, and CAVI did not differ between trials ( $p>0.05$, Fig. 2 ). The baPWV was higher at 30,60 and $90 \mathrm{~min}$ than at baseline after ingestion of $25 \mathrm{~g}$ glucose trial $(\mathrm{p}<0.05$, Fig. 2A), and significantly higher at 90 min than at baseline after ingestion of $20 \mathrm{~g}$ glucose trial ( $<<0.05$, Fig. $2 \mathrm{~A}$ ), but no significant changes were seen after ingestion of $15 \mathrm{~g}$ glucose trial $(\mathrm{p}>0.05$, Fig. 2A). The hbPWV was no different at 30,60 , or 90 min than at baseline after ingestion of any quantity of glucose ( $p>0.05$, Fig. 2B). CAVI was significantly higher at $60 \mathrm{~min}$ than at baseline after ingestion of $25 \mathrm{~g}$ glucose trial $(\mathrm{p}<0.05$, Fig. $2 \mathrm{C})$, but no significant differences were seen following the 15 or $20 \mathrm{~g}$ trial $(\mathrm{p}>0.05$, Fig. $2 \mathrm{C}$ ).

Brachial SBP, DBP and PP and ankle DBP did not differ significantly according to glucose dose ( $p>0.05$ each, Table 2). Brachial SBP, DBP and $\mathrm{PP}$ and ankle DBP were not significantly different at 30,60 or $90 \mathrm{~min}$ than at baseline after ingesting any quantity of glucose $(\mathrm{p}>0.05$ each, Table 2). Ankle SBP was significantly higher at 30,60 and $90 \mathrm{~min}$ than at baseline after ingestion of $25 \mathrm{~g}$ glucose trial $(\mathrm{p}<0.05$, Table 2), and at 60 and $90 \mathrm{~min}$ after ingestion of $20 \mathrm{~g}$ glucose trial $(\mathrm{p}<0.05$, Table 2$)$, but no significant differences were seen following the ingestion of $15 \mathrm{~g}$ glucose trial ( $\mathrm{p}>0.05$, Table 2 ). Ankle PP was significantly higher at 60 and 90 min than at baseline after ingestion of 20 and $25 \mathrm{~g}$ glucose trial ( $p<0.05$, Table 2$)$, but no significant differences were seen following ingestion of $15 \mathrm{~g}$ glucose trial ( $p>0.05$, Table 2).

Blood glucose was significantly higher at $60 \mathrm{~min}$ after the ingestion in the ingestion of 20 or $25 \mathrm{~g}$ glucose compared with the ingestion of $15 \mathrm{~g}$ glucose trial ( $p<0.05$, Table 3$)$. Compared to baseline, BG was significantly higher at $30 \mathrm{~min}$ after ingestion of $15 \mathrm{~g}$ glucose trial ( $\mathrm{p}<0.05$, Table 3$)$, at 30 and $60 \mathrm{~min}$ after ingestion of $20 \mathrm{~g}$ glucose trial $(\mathrm{p}<0.05$, Table 3 ), and at 30,60 , and $90 \mathrm{~min}$ after ingestion of $25 \mathrm{~g}(\mathrm{p}<0.05$, Table 3$)$. Compared to baseline, serum insulin was significantly higher at 


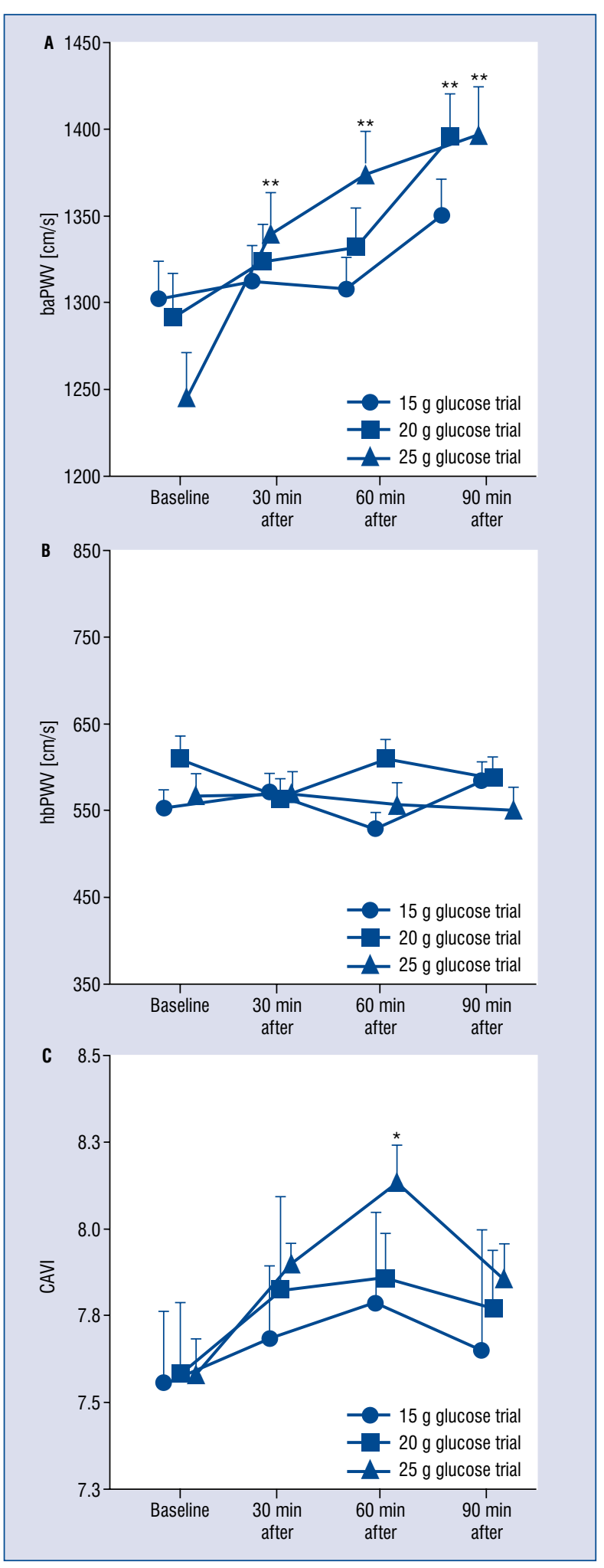

Figure 2. Changes in pulse wave velocity and cardio-ankle vascular index (CAVI) before (baseline) and after glucose ingestion. Values represent mean \pm standard deviation; baPWV - brachial-ankle pulse wave velocity; hbPWV - heart-brachial pulse wave velocity; ${ }^{*} p<0.05$ vs. baseline; ${ }^{* *} p<0.01$ vs. baseline.
30 min after ingestion of 15 g glucose trial $(\mathrm{p}<0.05$, Table 3), at 60 and $90 \mathrm{~min}$ after $20 \mathrm{~g}(\mathrm{p}<0.05$, Table 3), and at 30, 60, and $90 \mathrm{~min}$ than at baseline after $25 \mathrm{~g}$ glucose trial $(\mathrm{p}<0.05$, Table 3$)$.

\section{Discussion}

The novel finding of this study was that baPWV and CAVI were significantly increased after ingestion of $25 \mathrm{~g}$ glucose, and baPWV was significantly increased after ingestion of $20 \mathrm{~g}$ glucose, but not after ingestion of $15 \mathrm{~g}$. These results suggest that baPWV and CAVI only increase after larger doses of glucose. It was therefore proposed that glucose intake in a single meal should be reduced to prevent significant post-prandial increases in arterial stiffness.

Arterial stiffness acutely increases following high-carbohydrate meals. Indeed, Grassi et al. [14] and Gomez-Sanchez et al. [15] reported that systemic and peripheral arterial stiffness increased after glucose ingestion in healthy volunteers. Higher cardiovascular risk is associated with post-prandial hyperglycemia, which causes arterial stiffening [7]. The current WHO guideline recommends that adults and children reduce the daily intake of free sugars to $<25 \mathrm{~g}$ [8]. It was shown that baPWV was significantly higher at 30, 60 and 90 min than at baseline after ingestion of $25 \mathrm{~g}$ glucose, and was significantly higher at $90 \mathrm{~min}$ than at baseline after ingestion of $20 \mathrm{~g}$ glucose, whereas no significant change was seen after ingestion of $15 \mathrm{~g}$. Thus, consuming drinks and meals with lower sugar content might be effective in reducing the amount of glucose ingested at a single sitting, and thus suppress the increase in arterial stiffness that follows glucose ingestion. In addition, future research should use a method that does not increase arterial stiffness because of high-dose glucose ingestion, such as by changing from the use of glucose to palatinose.

Aortic arterial stiffness increases after glucose ingestion in obese individuals and those with metabolic syndrome [16]. It was previously reported that leg PWV increases acutely, whereas aortic PWV does not change, after oral ingestion of $75 \mathrm{~g}$ glucose in healthy individuals [5, 17]. Current findings indicate that both baPWV and CAVI significantly increase after ingestion of $25 \mathrm{~g}$ glucose, and baPWV significantly increases after ingestion of $20 \mathrm{~g}$ of glucose, whereas hbPWV (an indicator of proximal aortic stiffness) is unaffected by ingestion of $15-25 \mathrm{~g}$ glucose in healthy older individuals. 
Table 2. Changes in cardiovascular indices before and after glucose ingestion.

\begin{tabular}{|c|c|c|c|c|}
\hline & Baseline & After $30 \mathrm{~min}$ & After $60 \mathrm{~min}$ & After $90 \mathrm{~min}$ \\
\hline \multicolumn{5}{|c|}{ Brachial systolic blood pressure [mmHg] } \\
\hline 15 g glucose ingestion & $120 \pm 20$ & $118 \pm 12$ & $120 \pm 16$ & $122 \pm 17$ \\
\hline $20 \mathrm{~g}$ glucose ingestion & $118 \pm 16$ & $119 \pm 15$ & $123 \pm 14$ & $125 \pm 20$ \\
\hline 25 g glucose ingestion & $119 \pm 17$ & $120 \pm 15$ & $123 \pm 14$ & $121 \pm 17$ \\
\hline \multicolumn{5}{|c|}{ Brachial diastolic blood pressure [mmHg] } \\
\hline 15 g glucose ingestion & $76 \pm 15$ & $75 \pm 10$ & $76 \pm 10$ & $78 \pm 9$ \\
\hline 20 g glucose ingestion & $75 \pm 14$ & $75 \pm 11$ & $79 \pm 10$ & $80 \pm 13$ \\
\hline 25 g glucose ingestion & $75 \pm 15$ & $78 \pm 11$ & $76 \pm 11$ & $77 \pm 12$ \\
\hline \multicolumn{5}{|c|}{ Brachial pulse pressure [mmHg] } \\
\hline $15 \mathrm{~g}$ glucose ingestion & $44 \pm 6$ & $42 \pm 4$ & $43 \pm 8$ & $44 \pm 10$ \\
\hline 20 g glucose ingestion & $42 \pm 4$ & $44 \pm 7$ & $43 \pm 5$ & $45 \pm 8$ \\
\hline 25 g glucose ingestion & $44 \pm 4$ & $42 \pm 8$ & $46 \pm 5$ & $43 \pm 5$ \\
\hline \multicolumn{5}{|c|}{ Ankle systolic blood pressure [mmHg] } \\
\hline 15 g glucose ingestion & $141 \pm 11$ & $141 \pm 9$ & $141 \pm 9$ & $146 \pm 9$ \\
\hline 20 g glucose ingestion & $140 \pm 10$ & $144 \pm 10$ & $152 \pm 11^{* *}$ & $151 \pm 12^{* *}$ \\
\hline 25 g glucose ingestion & $139 \pm 11$ & $147 \pm 9 *$ & $153 \pm 10^{* *}$ & $151 \pm 10^{* *}$ \\
\hline \multicolumn{5}{|c|}{ Ankle diastolic blood pressure $[\mathrm{mmHg}]$} \\
\hline $15 \mathrm{~g}$ glucose ingestion & $76 \pm 15$ & $75 \pm 10$ & $76 \pm 11$ & $77 \pm 9$ \\
\hline 20 g glucose ingestion & $76 \pm 11$ & $76 \pm 13$ & $80 \pm 11$ & $80 \pm 13$ \\
\hline $25 \mathrm{~g}$ glucose ingestion & $76 \pm 12$ & $78 \pm 12$ & $78 \pm 10$ & $78 \pm 15$ \\
\hline \multicolumn{5}{|c|}{ Ankle pulse pressure [mmHg] } \\
\hline $15 \mathrm{~g}$ glucose ingestion & $65 \pm 13$ & $65 \pm 12$ & $64 \pm 15$ & $68 \pm 15$ \\
\hline $20 \mathrm{~g}$ glucose ingestion & $64 \pm 14$ & $68 \pm 16$ & $71 \pm 17^{*}$ & $71 \pm 17^{*}$ \\
\hline 25 g glucose ingestion & $62 \pm 16$ & $68 \pm 12$ & $74 \pm 16^{* *}$ & $72 \pm 11^{* *}$ \\
\hline \multicolumn{5}{|l|}{ Heart rate [beats/min] } \\
\hline 15 g glucose ingestion & $62 \pm 9$ & $60 \pm 8$ & $59 \pm 8$ & $62 \pm 10$ \\
\hline $20 \mathrm{~g}$ glucose ingestion & $63 \pm 9$ & $59 \pm 7$ & $60 \pm 7$ & $58 \pm 7$ \\
\hline 25 g glucose ingestion & $62 \pm 9$ & $60 \pm 10$ & $62 \pm 9$ & $59 \pm 8$ \\
\hline
\end{tabular}

Values are mean \pm standard deviation. ${ }^{*} p<0.05$ vs. baseline; ${ }^{* *} p<0.01$ vs. baseline

Table 3. Changes in blood glucose and insulin levels before and after glucose ingestion.

\begin{tabular}{|c|c|c|c|c|}
\hline & Baseline & After $30 \mathrm{~min}$ & After $60 \mathrm{~min}$ & After $90 \mathrm{~min}$ \\
\hline \multicolumn{5}{|l|}{ Blood glucose $[\mathrm{mg} / \mathrm{dL}]$} \\
\hline 15 g glucose ingestion & $94 \pm 9$ & $130 \pm 28^{* *}$ & $108 \pm 17$ & $94 \pm 17$ \\
\hline $20 \mathrm{~g}$ glucose ingestion & $98 \pm 5$ & $139 \pm 21 * *$ & $137 \pm 26^{* *, \dagger}$ & $110 \pm 20$ \\
\hline 25 g glucose ingestion & $93 \pm 12$ & $142 \pm 21 * *$ & $137 \pm 26^{* *,+}$ & $107 \pm 20^{* *}$ \\
\hline \multicolumn{5}{|l|}{ Insulin $[\mathrm{mg} / \mathrm{dL}]$} \\
\hline $15 \mathrm{~g}$ glucose ingestion & $5 \pm 3$ & $11 \pm 9 *$ & $9 \pm 5$ & $6 \pm 5$ \\
\hline $20 \mathrm{~g}$ glucose ingestion & $5 \pm 2$ & $10 \pm 4$ & $15 \pm 8^{* *}$ & $9 \pm 2^{* *}$ \\
\hline 25 g glucose ingestion & $5 \pm 2$ & $13 \pm 8^{*}$ & $13 \pm 5^{* *}$ & $9 \pm 2^{* *}$ \\
\hline
\end{tabular}

Values represent mean \pm standard deviation. ${ }^{*} p<0.05$ vs. baseline; ${ }^{* *} p<0.01$ vs. baseline; $\uparrow p<0.05$ vs. 15 g glucose ingestion

The increase in arterial stiffness after ingestion of glucose might thus be stronger in the lower limb artery than in the aorta. However, changes in aortic PWV after glucose ingestion might occur in obese older individuals (those with high insulin resistance). 
Greater arterial stiffness after meals is associated with increases in SBP $[5,18]$. It was shown herein, that hbPWV and brachial SBP were not significantly changed after ingestion of glucose. The present study shows that baPWV and ankle SBP increased after ingestion of glucose. It was previously reported that leg PWV and ankle SBP increased acutely, whereas aortic PWV and aortic SBP did not change significantly, after oral ingestion of $75 \mathrm{~g}$ glucose in healthy individuals $[5,18]$. This rise in ankle SBP may be indicative of an increase in baPWV (the main is leg PWV) after ingestion of 20 and $25 \mathrm{~g}$ glucose. However, this was not directly assessed, representing an important limitation of this study. Importantly, it was shown that CAVI increased significantly after ingestion of $25 \mathrm{~g}$ glucose. CAVI is independent of blood pressure variations during measurement [19]. The level of oxidative stress, as determined by measuring thiobarbituric acid reactive substances (TBARS), acutely increases after a 75 -g oral glucose tolerance test [20], and Kawano et al. [20] have shown that TBARS after glucose ingestion correlates with BG. Tso et al. [21] have shown that TBARS correlates with arterial stiffness. Thus, the increase in CAVI after ingesting $25 \mathrm{~g}$ glucose might be associated with oxidative stress induced by hyperglycemia. However, levels of oxidative stress were not assessed, representing another limitation of the present study.

From the above context, it was considered that increased arterial stiffness after ingesting $20 \mathrm{~g}$ of glucose was mainly related to elevated SBP. Moreover, increased arterial stiffness after ingesting $25 \mathrm{~g}$ of glucose might be associated with decreased vascular endothelial function due to increased oxidative stress and a rise in SBP. Studying the mechanisms underlying increases in arterial stiffness due to differences in glucose intake will be important in future research.

\section{Limitations of the study}

Some other limitations must be addressed when considering the present findings. The participants were healthy subjects, which precludes the generalization of our findings to younger individuals. In addition, the sample size was very small $(\mathrm{n}=6)$, although the effects of glucose dose on arterial stiffness differed sufficiently in detecting significant differences and the differences identified were similar to those identified in previous studies $(n=6)$ of acute changes in arterial stiffness [21]. There was also no control group. A further limitation is the lack of carotid-femoral PWV which is the gold standard technique for the assessment of arterial stiffness. Finally, this study was not designed to determine the possible mechanisms underlying the acute effects of glucose intake on PWV and CAVI.

\section{Conclusions}

Significant increases in baPWV and CAVI were identified after ingestion of $25 \mathrm{~g}$ glucose, and baPWV significantly increased after ingestion of $20 \mathrm{~g}$ glucose, but neither increased after $15 \mathrm{~g}$ of glucose. This suggests that arterial stiffness is only acutely affected by the ingestion of larger quantities of glucose. It was therefore proposed that glucose intake should be reduced at each meal to avoid increases in arterial stiffness during acute hyperglycemia.

\section{Acknowledgements}

This work was supported by a research funding from Mitsui Sugar Co., Ltd.

\section{Conflict of interest: None declared}

\section{References}

1. Yamashina A, Tomiyama H, Arai T, et al. Nomogram of the relation of brachial-ankle pulse wave velocity with blood pressure. Hypertens Res. 2003; 26(10): 801-806, doi: 10.1291/hypres.26.801, indexed in Pubmed: 14621183.

2. van Sloten TT, Schram MT, van den Hurk K, et al. Local stiffness of the carotid and femoral artery is associated with incident cardiovascular events and all-cause mortality: the Hoorn study. J Am Coll Cardiol. 2014; 63(17): 1739-1747, doi: 10.1016/j. jacc.2013.12.041, indexed in Pubmed: 24583306.

3. Tsuchikura S, Shoji T, Kimoto E, et al. Central versus peripheral arterial stiffness in association with coronary, cerebral and peripheral arterial disease. Atherosclerosis. 2010; 211(2): 480-485, doi: 10.1016/j.atherosclerosis.2010.03.037, indexed in Pubmed: 20430390.

4. Wohlfahrt P, Krajčoviechová A, Seidlerová J, et al. Lowerextremity arterial stiffness vs. aortic stiffness in the general population. Hypertens Res. 2013; 36(8): 718-724, doi: 10.1038/ hr.2013.21, indexed in Pubmed: 23575382.

5. Kobayashi R, Yoshida S, Okamoto T. Arterial stiffness after glucose ingestion in exercise-trained versus untrained men. Appl Physiol Nutr Metab. 2015; 40(11): 1151-1156, doi: 10.1139/ apnm-2015-0131, indexed in Pubmed: 26444929.

6. Kobayashi R, Yoshida S, Okamoto T. Effects of acute aerobic exercise on arterial stiffness before and after glucose ingestion. Int J Sports Med. 2017; 38(1): 12-18, doi: 10.1055/s-0042-115019, indexed in Pubmed: 27813047.

7. Jacome-Sosa M, Parks EJ, Bruno RS, et al. Postprandial metabolism of macronutrients and cardiometabolic risk: recent developments, emerging concepts, and future directions. Adv Nutr. 2016; 7(2): 364-374, doi: 10.3945/an.115.010397, indexed in Pubmed: 26980820 . 
8. WHO Guidelines Approved by the Guidelines Review Committee. Guideline: sugars intake for adults and children. World Health Organization. Geneva 2015.

9. Jenkins DJ, Wolever TM, Taylor RH, et al. Glycemic index of foods: a physiological basis for carbohydrate exchange. Am J Clin Nutr. 1981; 34(3): 362-366, doi: 10.1093/ajcn/34.3.362, indexed in Pubmed: 6259925.

10. Sugawara J, Tomoto T, Tanaka H. Heart-to-Brachium pulse wave velocity as a measure of proximal aortic stiffness: MRI and longitudinal studies. Am J Hypertens. 2019; 32(2): 146-154, doi: 10.1093/ajh/hpy166, indexed in Pubmed: 30445561.

11. Nishiwaki M, Takahara K, Matsumoto N. Arterial stiffness in young adult swimmers. Eur J Appl Physiol. 2017; 117(1): 131-138, doi: 10.1007/s00421-016-3505-9, indexed in Pubmed: 27873019.

12. Okamoto T, Sakamaki MS, Min SK, et al. Repeated cessation and resumption of resistance training attenuates increases in arterial stiffness. Int J Sports Med. 2015; 36(6): 440-445, doi: 10.1055/s0034-1398584, indexed in Pubmed: 25734911.

13. Seino Y, Nanjo K, Tajima N, et al. Report of the committee on the classification and diagnostic criteria of diabetes mellitus. J Diabetes Investig. 2010; 1(5): 212-228, doi: 10.1111/j.20401124.2010.00074.x, indexed in Pubmed: 24843435.

14. Grassi D, Desideri G, Necozione S, et al. Protective effects of flavanol-rich dark chocolate on endothelial function and wave reflection during acute hyperglycemia. Hypertension. 2012; 60(3): 827-832, doi: 10.1161/HYPERTENSIONAHA.112.193995, indexed in Pubmed: 22851734.
15. Gomez-Sanchez L, Garcia-Ortiz L, Patino-Alonso MC, et al. MARK Group. Glycemic markers and relation with arterial stiffness in Caucasian subjects of the MARK study. PLoS One. 2017; 12(4): e0175982, doi: 10.1371/journal.pone.0175982, indexed in Pubmed: 28414819.

16. Baynard T, Carhart RL, Weinstock RS, et al. Short-term exercise training improves aerobic capacity with no change in arterial function in obesity. Eur J Appl Physiol. 2009; 107(3): 299-308, doi: 10.1007/s00421-009-1126-2, indexed in Pubmed: 19609554.

17. Kobayashi R, Hashimoto Y, Hatakeyama H, et al. Acute effects of aerobic exercise intensity on arterial stiffness after glucose ingestion in young men. Clin Physiol Funct Imaging. 2018; 38(1): 138-144, doi: 10.1111/cpf.12395, indexed in Pubmed: 27758064.

18. Blendea MC, Bard M, Sowers JR, et al. High-fat meal impairs vascular compliance in a subgroup of young healthy subjects. Metabolism. 2005; 54(10): 1337-1344, doi: 10.1016/j.metabol.2005.04.023, indexed in Pubmed: 16154433.

19. Shirai K, Utino J, Otsuka K, et al. A novel blood pressure-independent arterial wall stiffness parameter; cardio-ankle vascular index (CAVI). J Atheroscler Thromb. 2006; 13(2): 101-107, doi: 10.5551/jat.13.101, indexed in Pubmed: 16733298.

20. Kawano H, Motoyama T, Hirashima O, et al. Hyperglycemia rapidly suppresses flow-mediated endothelium-dependent vasodilation of brachial artery. J Am Coll Cardiol. 1999; 34(1): 146-154, doi: 10.1016/s0735-1097(99)00168-0.

21. Tso TK, Huang WN, Huang HY, et al. Association of brachialankle pulse wave velocity with cardiovascular risk factors in systemic lupus erythematosus. Lupus. 2005; 14(11): 878-883, doi: 10.1191/0961203305lu2234oa, indexed in Pubmed: 16335579. 